\title{
Control design of a de-weighting upper limb exoskeleton
}

\begin{abstract}
One of the most common problems in humans is a muscle fatigue. Exoskeletons are known as one of the solution to deal with human muscle fatigue. However, several issues related to the development of exoskeletons for such a case have been identified. One of these is the control mechanism. Thus, the objective of this paper is to investigate development of a control strategy for the upper-limb exoskeleton. In this paper, a new control mechanism for an upperlimb exoskeleton is proposed. A fuzzy-based PD controller and PID are used in the proposed control mechanism, and a comparative assessment of the performance of both controllers is made. The results show that the control mechanism with fuzzy-based PD controller performs better than the PID controller in terms of trajectory tracking accuracy and control torque analysis.
\end{abstract}

Keyword: Exoskeleton; Kinematics and dynamics; Euler-Lagrange approach; Fuzzy-based control 